\title{
Clinical significance of ectopic inner foveal layers in patients with idiopathic epiretinal membranes
}

\author{
Sibel Doguizi ${ }^{1} \cdot$ Mehmet Ali Sekeroglu$^{1} \cdot$ Dilara Ozkoyuncu $^{1} \cdot$ Aslihan Esra Omay $^{1} \cdot$ Pelin Yilmazbas $^{1}$
}

Received: 3 February 2018 / Revised: 6 May 2018 / Accepted: 12 May 2018 / Published online: 22 June 2018

(c) The Royal College of Ophthalmologists 2018

\begin{abstract}
Background/Objectives To evaluate the relationship between the presence of ectopic inner foveal layers (EIFL), choroidal thickness, and visual acuity (VA) in patients with epiretinal membranes (ERM) staged by spectral-domain optical coherence tomography (SD-OCT).

Subjects/Methods A total of 242 eyes of 121 patients with unilateral idiopathic ERM were prospectively evaluated. ERM stages were defined based on the SD-OCT staging system as stage 1: negligible morphological or anatomical disruption, retinal layers, and foveal pit are identified; stage 2: characteristic stretching of the outer nuclear layer, absence of foveal depression, retinal layers are identified; stage 3: continuous EIFL crossing the central foveal area, absence of foveal depression, retinal layers are identified; and stage 4: anatomical disruption of the fovea, continuous EIFL crossing the entire foveal area, retinal layers are distorted.

Results Of 121 eyes with ERM, 23.1\% had stage 1, 26.5\% had stage 2, 39.7\% had stage 3, and $10.7 \%$ had stage 4 disease. VA was better in eyes with stage 1 or 2 ERM than stage 3 or 4 ERM $(p<0.001)$. VA in logMAR was positively correlated with central foveal thickness $(r=0.557, p<0.001)$ and EIFL thickness $(r=0.526, p<0.001)$, but not with an outer nuclear layer thickness $(r=0.233, p=0.123)$. In multivariate analysis, the presence of EIFL was an independent predictor of VA in eyes with ERM $(p<0.001)$. The presence and stage of ERM did not have a significant effect on choroidal thickness $(p>$ $0.05)$.

Conclusions The SD-OCT staging system according to the presence of EIFL is effective for grading retinal damage and visual loss in eyes with ERM.
\end{abstract}

\section{Introduction}

Epiretinal membranes (ERMs) are avascular fibrous layers characterized by abnormal fibrocellular proliferation on the inner retinal surface with a prevalence ranging from 2.2 to $28.9 \%$, and they are more common in elderly people [1-4]. ERM involving the macular or peri-macular regions can cause visual impairment, metamorphopsia, micropsia, and occasionally monocular diplopia. Although ERMs may develop secondary to cataract surgery, retinal detachment, retinal vein occlusions, diabetic

Sibel Doguizi

eryigits@yahoo.com

1 Department of Ophthalmology, Ulucanlar Eye Training and Research Hospital, Ankara, Turkey retinopathy, or uveitis and retinal tearing, most cases are idiopathic [5-7].

ERMs were initially diagnosed and classified based on clinical findings alone [8]. More recently, imaging techniques, such as spectral-domain optical coherence tomography (SD-OCT) with three-dimensional reconstruction, have been introduced for this purpose. With the introduction of SD-OCT, clinicians can also evaluate microstructural changes of the macula region both qualitatively and quantitatively, which is needed to assess the severity of ERM and to predict possible vision loss [9-11]. Many reports have demonstrated a relationship between vision loss associated with ERM and disruption of the ellipsoid zone and outer photoreceptor segments [12-15]. The visual loss associated with ERM has also been suggested to be caused by retinal ganglion or inner nuclear layer cell damage due to the inner retinal layer wrinkling, which is represented as an increase in inner-retinal thickness or ganglion cell-inner plexiform layer thickness [16-19]. A 
role of the choroid in the development and prognosis of ERM has also been proposed by some studies, but has not yet been clarified [20, 21].

In order to fully harness SD-OCT in diagnosing and assessing the severity of ERM, a globally accepted OCTbased staging system that takes into account findings related to vision loss is lacking. Govetto et al. [22] recently suggested that the presence of continuous ectopic inner foveal layers (EIFL) in ERMs in OCT is associated with significant vision loss and is thus an essential element of a novel OCT-based staging scheme of ERMs. However, the practical use of this novel staging system has not yet been evaluated by other studies.

In this study, we aimed to investigate this new OCTbased staging system in our ERM patients and to evaluate the microstructural SD-OCT findings of the macula, choroidal thickness, and visual acuity in different stages of ERM.

\section{Methods}

\section{Study population}

This was a prospective cross-sectional study in which 242 consecutive eyes of 121 patients (mean age: 69.7 \pm 7.3 years, male to female ratio: 62:59) with unilateral idiopathic ERM were evaluated in a single institution between December 2016 and December 2017. The exclusion criteria were secondary ERM (due to diabetic retinopathy, venous occlusion, retinal tear, retinal detachment, uveitis, trauma); eyes with pseudoholes, lamellar holes, or opaque media; glaucoma; history of intraocular surgery (except uncomplicated cataract surgery); uncontrolled diabetes or hypertension; coexisting age-related macular degeneration; nicotine abuse; and refractive errors above -2 and +2 diopters.

This study was approved by the Institutional Review Board of the Numune Eye Training and Research Hospital (No. E-17-1685) and conducted according to the tenets of the Declaration of Helsinki. Patients gave written informed consent before the study procedures.

\section{Ophthalmologic evaluation}

All patients underwent a comprehensive ophthalmologic examination, including measurement of best corrected visual acuity (BCVA) using a Snellen chart, indirect slitlamp biomicroscopy and intraocular pressure measurements using a non-contact tonometer (Nidek NT-530P, Nidek Co. Ltd., Aichi, Japan). BCVA was recorded in terms of Snellen fractions and converted into logarithm of the minimal angle of resolution (logMAR) values.

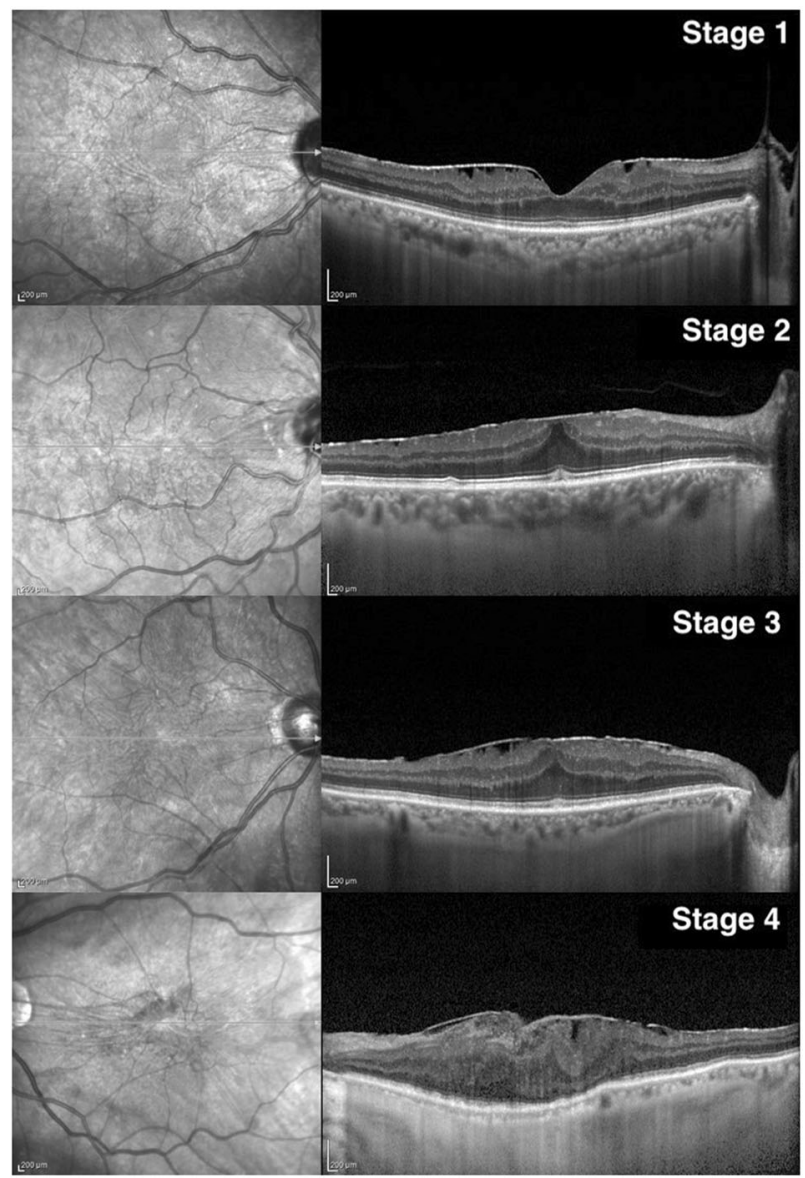

Fig. 1 OCT images of epiretinal membranes according to the staging system by Govetto et al. [22]. Stage 1: negligible morphological or anatomical disruption, retinal layers, and foveal pit are identified; Stage 2: characteristic stretching of the outer nuclear layer, absence of foveal depression, retinal layers are identified; Stage 3: continuous ectopic inner foveal layers crossing the central foveal area, absence of foveal depression, retinal layers are identified; Stage 4: significant retinal thickening, remarkable anatomical disruption of the macula, continuous ectopic inner foveal layers crossing the entire foveal area, retinal layers are significantly distorted, and foveal pit is absent

\section{SD-OCT imaging and quantitative measurements}

OCT images were obtained with the Spectralis OCT with eye-tracking dual-beam technology (Heidelberg Engineering $\mathrm{GmbH}$, Heidelberg, Germany), and SD-OCT images were generated using the horizontal spectral-domain OCT cross-section (25 lines spaced $240 \mathrm{~mm}$ apart; $20^{\circ} \times 20^{\circ}$ ).

Spectralis OCT scan patterns were used for all quantitative measurements. We classified our patients according to the OCT-based staging system devised by Govetto et al. [22] (Fig. 1). The microstructural SD-OCT findings of the macula including microcystoid retinal changes, presence of the cotton ball sign, and disruption of the ellipsoid zone were also evaluated.

Continuous EIFL was defined on OCT as the presence of a continuous hypo- or hyper-reflective band, extending from 
the inner nuclear layer and inner plexiform layer across the foveal region, as described before by Govetto et al. [22]. Disruption of the inner segment ellipsoid zone was defined as a discontinuous ellipsoid band in the foveal region, and the presence of the cotton ball sign was defined as a round or diffuse hyper-reflective area between the ellipsoid zone and the cone outer segment tip line at the central fovea, as described by Tsunoda et al. [23]. The microcystoid retinal changes were described as the presence of multiple, small hypo-reflective roundish-elliptical cystoid spaces in retinal layers.

The central foveal thickness was measured with the automated thickness function of the Heidelberg Eye Explorer, and the thicknesses of EIFL and the outer nuclear layer in the foveal region were measured manually with the "caliper" function of the Heidelberg instrument.

The thickness of EIFL could be measured only in 48 eyes with ERM, since EIFL was present in eyes with stage 3 or stage 4 ERM, and the retinal layers in stage 4 could not be distinguished. Outer nuclear layer thickness was measured in eyes with stage 1, 2, or 3 ERM, but not in eyes with stage 4 ERM.

\section{EDI-OCT imaging and choroidal thickness measurements}

For choroidal thickness measurements, choroidal imaging was performed using the enhanced depth imaging (EDI) mode of the SD-OCT (Heidelberg Engineering $\mathrm{GmbH}$, Heidelberg, Germany), which automatically reinverts the inverted images and enables EDI-OCT images to be captured directly. The imaging was performed while the patient was seated in a resting position at the same time of the day between 3 p.m. and 6 p.m. Subfoveal choroidal thickness was measured from the outer surface of the hyper-reflective line ascribed to the retinal pigment epithelium to the hyper-reflective line of the inner sclera border. The section going directly through the center of the fovea was selected for this measurement. The choroidal thickness was manually measured at five points (subfoveal, temporal and nasal at 1000 and $2000 \mu \mathrm{m}$ ) with the "caliper" function of the Heidelberg instrument. The measurement was performed three times, and the average was used as the result. The choroidal thicknesses of eyes with ERM were determined with respect to stage of ERM and were also compared with the measurements of healthy fellow eyes.

For precise qualitative and quantitative analyses, all parameters were measured separately and independently by two retina specialists (SD, MAS) who were masked to the patients' clinical details. If there were any discrepancies in the measurement of qualitative parameters, another investigator (PY) was consulted for the final decision.

\section{Statistical analysis}

All patients who met the study criteria were included in the study during the recruitment period. Descriptive statistics are expressed as mean \pm standard deviation or median (minimum-maximum) for continuous variables and as frequency and percentage for categorical variables. The normal distribution of the continuous variables was tested using the Kolmogorov-Smirnov test, and the homogeneity of variance was tested using the Levene test. The data show a non-normal distribution, and the variance between groups of ERM stages was not similar. Therefore, non-parametric tests were used for the analysis. The significance of the difference between the independent groups was examined with the Mann-Whitney $U$ test for two groups and with the Kruskal-Wallis test for more than two groups. The analysis of categorical data in cross tables was performed using the likelihood ratio test or the Fisher's exact test if any expected count was less than 5; otherwise, it was performed using the Pearson's Chi-square test. The choroidal thicknesses of the eyes with ERM and those of healthy fellow eyes were compared using the Wilcoxon sign test. The significance of the relationship between continuous variables was investigated using the Spearman's rank correlation test. The multivariate linear regression analysis was used to determine the most significant independent predictor of visual acuity in the ERM eyes. All the variables with $p<0.10$ in univariate analyses were included in the multivariable linear regression model.

Analysis of the data was performed using IBM SPSS Statistics 17.0 software (IBM Corporation, Armonk, NY, USA). The results were considered statistically significant when $p$ was less than 0.05 .

\section{Results}

According to the SD-OCT-based staging system, out of 121 eyes, 28 (23.1\%) had stage 1, $32(26.5 \%)$ had stage 2, 48 (39.7\%) had stage 3, and $13(10.7 \%)$ had stage 4 ERM (Tables 1 and 2). ERM was localized in the right eye in 66 patients $(54.5 \%)$. The median BCVA of all eyes with ERM was $0.4 \log$ MAR (range: $0.0-1.3 \log \mathrm{MAR}$ ). The BCVA was significantly better in eyes with stage 1 or stage 2 ERM than in eyes with stage 3 or stage 4 ERM $(p<0.001$, Table 1). The intraocular pressures of 121 eyes with ERM were within normal limits (mean: $14.0 \pm 2.3 \mathrm{mmHg}$, range: 8-20 mmHg).

\section{SD-OCT findings}

On SD-OCT, microcystoid retinal changes were recorded in 30 out of 121 eyes $(24.7 \%)$, the cotton ball sign in 23 eyes 
Table 1 SD-OCT findings with respect to the stage of epiretinal membranes

\begin{tabular}{|c|c|c|c|c|c|}
\hline & Stage $1(n=28)$ & Stage $2(n=32)$ & Stage $3(n=48)$ & Stage $4(n=13)$ & $p$ Value \\
\hline BCVA (logMAR) & $\begin{array}{l}0.2 \\
(0.0-0.5)\end{array}$ & $\begin{array}{l}0.2 \\
(0-1.0)\end{array}$ & $\begin{array}{l}0.4 \\
(0.1-1.3)\end{array}$ & $\begin{array}{l}0.6 \\
(0.3-1.3)\end{array}$ & $<0.001^{\mathrm{a}}$ \\
\hline $\begin{array}{l}\text { Microcystoid retinal } \\
\text { changes }\end{array}$ & $4(14.2 \%)$ & $7(21.8 \%)$ & $12(25 \%)$ & $7(53.8 \%)$ & $0.395^{\mathrm{b}}$ \\
\hline Cotton ball sign & $2(7.1 \%)$ & $9(28.1 \%)$ & $12(25 \%)$ & $0(0.0)$ & $0.004^{\mathrm{c}}$ \\
\hline $\begin{array}{l}\text { Disruption of ellipsoid } \\
\text { zone }\end{array}$ & $1(3.5 \%)$ & $3(9.3 \%)$ & $10(20.8 \%)$ & $8(61.5 \%)$ & $<0.001^{\mathrm{c}}$ \\
\hline $\begin{array}{l}\text { Central foveal thickness } \\
(\mu \mathrm{m})\end{array}$ & $\begin{array}{l}236 \\
(203-287)\end{array}$ & $\begin{array}{l}339.5 \\
(221-530)\end{array}$ & $\begin{array}{l}459.5 \\
(302-692)\end{array}$ & $\begin{array}{l}565 \\
(305-678)\end{array}$ & $<0.001^{\mathrm{a}}$ \\
\hline $\begin{array}{l}\text { Outer nuclear layer } \\
\text { thickness }(\mu \mathrm{m})\end{array}$ & $\begin{array}{l}117.5 \\
(53-190)\end{array}$ & $\begin{array}{l}204 \\
(101-347)\end{array}$ & $\begin{array}{l}177.5 \\
(84-323)\end{array}$ & - & $<0.001^{\mathrm{a}}$ \\
\hline $\begin{array}{l}\text { Ectopic inner foveal layer } \\
(\mu \mathrm{m})\end{array}$ & - & - & $\begin{array}{l}152.5 \\
(41-368)\end{array}$ & - & - \\
\hline
\end{tabular}

Data are presented as median (min-max) or $n(\%)$

${ }^{a}$ Kruskal-Wallis test

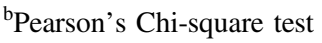

${ }^{c}$ Likelihood ratio test
(19.0\%), and disruption of the ellipsoid zone in 22 eyes (18.1\%). There was no statistically significant difference in terms of the rates of cystoid changes among ERM stages $(p=0.395)$. However, as the stages advanced, the rate of cystoid changes increased remarkably (Table 1). There was a statistically significant difference between the ERM stages in terms of the incidence of the cotton ball sign ( $p=0.004$, Table 1). The cotton ball sign was significantly more common in eyes with stage 2 and stage 3 ERM than in those with stage $1 \mathrm{ERM}$ ( $p=0.021$ and 0.01 , respectively). There was no evidence of the cotton ball sign in eyes with stage 4 ERM (Table 1). The disruption of the ellipsoid zone was significantly more prevalent in eyes with stage 3 and particularly stage 4 ERM than in eyes with earlier stages of the disease $(p<0.001$, Table 1$)$.

The median central foveal thickness, outer nuclear layer thickness, and EIFL thickness of eyes with ERM were measured as 389,170 , and $152.5 \mu \mathrm{m}$, respectively. The median central foveal thickness in the healthy fellow eyes of patients with ERM was $228 \mu \mathrm{m}$ (range: 206-282). The median central foveal thickness of eyes with ERM was significantly higher than that of healthy fellow eyes without ERM (389 vs. $228 \mu \mathrm{m}, p<0.001)$. The median central foveal thickness of healthy fellow eyes without ERM in patients with stage 1 ERM was measured as $226 \mu \mathrm{m}$ (range: $208-265$ ); stage $2 \mathrm{ERM}$ was measured as $230 \mu \mathrm{m}$ (range: 206-276); stage 3 ERM was measured as $231 \mu \mathrm{m}$ (range: $210-271$ ); and stage 4 ERM was measured as $225 \mu \mathrm{m}$ (range: $211-282$ ). The median central foveal thickness of eyes with ERM was significantly higher than that of healthy fellow eyes without ERM in patients with stage 1 ( 236 vs. $226 \mu \mathrm{m}, p=0.03$ ), stage 2 (339.5 vs.
$228 \mu \mathrm{m}, p<0.001$ ), stage 3 (459.5 vs. $231 \mu \mathrm{m}, p<0.001$ ), and stage 4 ERM (565 vs. $225 \mu \mathrm{m}, p<0.001$ ).

Significant increases were noted in central foveal thickness as the stage of ERM progressed $(p<0.001$ for all, Table 1). The central foveal thickness of eyes with stage 3 or 4 ERM was statistically higher than that of eyes with stage 1 or 2 ERM $(p<0.001)$. The outer nuclear layer thickness of eyes with stage 2 or stage 3 ERM was statistically higher than that of eyes with stage 1 ERM $(p<0.001$, Table 1$)$. There was no statistically significant difference between the outer nuclear layer thicknesses of eyes with stage 2 and stage 3 ERM $(p=0.071)$.

\section{BCVA with respect to SD-OCT findings}

The presence of microcystoid retinal changes or the cotton ball sign had no significant effect on BCVA $(p=0.548$ and 0.861 , respectively). However, eyes with EIFL or disruption of the ellipsoid zone were associated with significantly higher BCVA values in logMAR $(p<0.001$ and $p=0.009$, respectively) (Table 2).

BCVA in $\log$ MAR was positively correlated with the central foveal thickness of all eyes $(r=0.557, p<0.001)$ and the EIFL thickness of eyes with stage 3 ERM $(r=0.526, p<0.001)$, but not with outer nuclear layer thickness ( $r=0.233, p=0.123)$ (Fig. 2).

In multivariate linear regression analysis, controlling for other possible causes of reduced BCVA, central foveal thickness, and the presence of EIFL were independent predictive factors for lower visual acuity in eyes with ERM $(p<0.001$, for both). 
Table 2 Best corrected visual acuity (BCVA) with respect to SD-OCT findings

\begin{tabular}{lllll}
\hline & & $n$ & $\begin{array}{l}\text { BCVA } \\
(\operatorname{logMAR})\end{array}$ & $\begin{array}{l}p \\
\text { Value }^{\mathrm{a}}\end{array}$ \\
\hline Microcystoid retinal changes & Not present & 75 & $0.3(0.0-1.3)$ & 0.548 \\
& Present & 46 & $0.4(0.1-0.7)$ & \\
Ectopic inner foveal layer & Not present & 60 & $0.3(0.0-0.7)$ & $<0.001$ \\
& Present & 61 & $0.5(0.3-1.3)$ & \\
Cotton ball sign & Not present & 98 & $0.4(0.0-1.3)$ & 0.861 \\
& Present & 23 & $0.2(0.1-0.7)$ & \\
Disruption of ellipsoid zone & Not present & 99 & $0.3(0.0-1.3)$ & 0.009 \\
& Present & 22 & $0.5(0.2-1.3)$ & \\
\hline
\end{tabular}

Data are presented as median $(\min -\max )$

${ }^{a}$ Mann-Whitney $U$ test
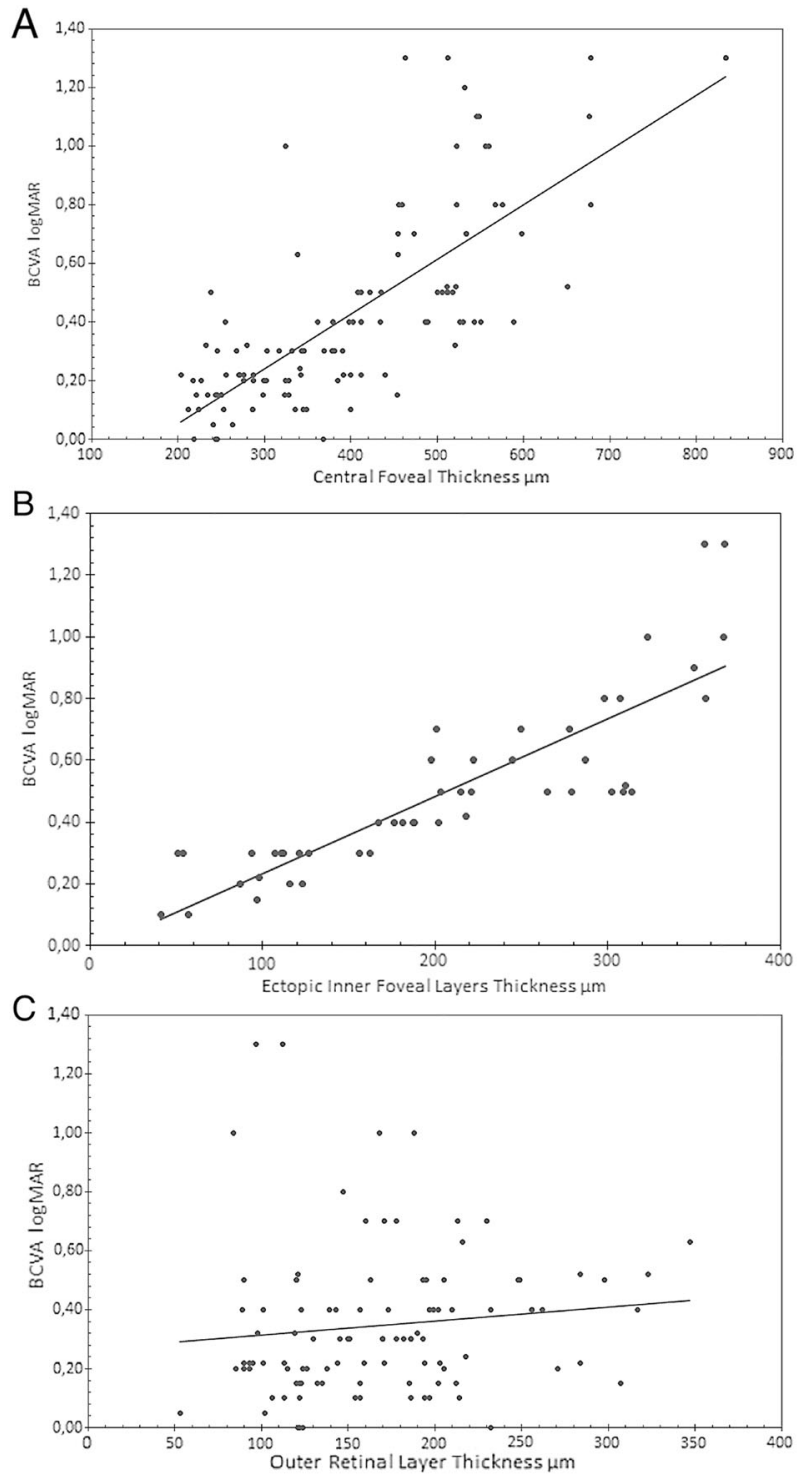

Fig. 2 Scattered diagrams showing a positive correlation between central foveal thickness and BCVA $(r=0.557, p<0.001)$ (a) and between ectopic inner foveal layer thickness and BCVA $(r=0.526, p$ $<0.001)(\mathbf{b})$, but no correlation between outer nuclear layer thickness and BCVA $(r=0.233, p=0.123)(\mathbf{c})$

\section{Choroidal thickness with respect to the presence and stage of ERM}

There was no statistically significant difference between eyes with ERM and healthy fellow eyes without ERM in terms of choroidal thickness obtained from the nasal, temporal, and subfoveal regions of eyes $(p>0.05$, Table 3$)$. Similarly, there was no significant difference between stages of ERM in terms of the choroidal thicknesses of the N2000, N1000, subfoveal, T1000, and T2000 regions ( $p>$ 0.05 , for all, Table 3 ).

\section{Discussion}

ERMs are common ocular abnormalities causing vision loss with a general prevalence of $1.1-18.5 \%$, which is significantly higher in the elderly population [2, 24]. Staging and understanding which layer's thickening is correlated with visual impairment is important to determine the indications and timing of vitrectomy for the treatment of eyes with ERM. In the present study, we primarily found that the SD-OCT-based staging system recently developed by Govetto et al. [22] is practical and effective for grading macular microstructural changes and visual loss in eyes with ERM.

Various staging systems have been proposed, on which no common consensus has been determined. Hwang et al. [25] classified idiopathic ERM into five subtypes according to the characteristics of foveal morphology on SD-OCT: outer retinal thickening with minimal inner retinal change, outer retinal inward projection with inner retinal thickening, prominent inner retinal thickening, formation of a macular pseudohole, and fovea-sparing ERM with schisis-like intraretinal splitting. They used multifocal electroretinography for the assessment of the functional differences and pathophysiology of different stages of ERM. Kinoshita et al. [26] categorized eyes with ERM into four 
Table 3 Comparison between subfoveal choroidal thickness $(\mu \mathrm{m})$ of nasal, temporal, and subfoveal regions of eyes with ERM and healthy fellow eyes without ERM, and according to the stage of ERM

\begin{tabular}{|c|c|c|c|c|c|c|c|c|}
\hline & $\begin{array}{l}\text { Eyes with } \\
\text { ERM } \\
(n=121)\end{array}$ & $\begin{array}{l}\text { Eyes } \\
\text { without } \\
\text { ERM } \\
(n=121)\end{array}$ & $p$ Value $^{\mathrm{a}}$ & $\begin{array}{l}\text { Stage } 1 \\
(n=26)\end{array}$ & $\begin{array}{l}\text { Stage } 2 \\
(n=32)\end{array}$ & $\begin{array}{l}\text { Stage } 3 \\
(n=48)\end{array}$ & $\begin{array}{l}\text { Stage } 4 \\
(n=13)\end{array}$ & $p$ Value $^{\mathrm{a}}$ \\
\hline N2000 & $\begin{array}{l}172.5 \\
(46-403)\end{array}$ & $\begin{array}{l}167 \\
(59-345)\end{array}$ & 0.332 & $\begin{array}{l}162.5 \\
(106-369)\end{array}$ & $\begin{array}{l}176 \\
(65-342)\end{array}$ & $\begin{array}{l}175.5 \\
(46-403)\end{array}$ & $\begin{array}{l}172.1 \\
(49-413)\end{array}$ & 0.953 \\
\hline N1000 & $\begin{array}{l}199 \\
(89-444)\end{array}$ & $\begin{array}{l}223 \\
(75-434)\end{array}$ & 0.052 & $\begin{array}{l}197 \\
(117-441)\end{array}$ & $\begin{array}{l}203.5 \\
(89-378)\end{array}$ & $\begin{array}{l}196 \\
(98-444)\end{array}$ & $\begin{array}{l}204 \\
(89-387)\end{array}$ & 0.738 \\
\hline Subfoveal & $\begin{array}{l}229.4 \\
(114-475)\end{array}$ & $\begin{array}{l}243 \\
(88-457)\end{array}$ & 0.091 & $\begin{array}{l}236 \\
(120-465)\end{array}$ & $\begin{array}{l}242 \\
(114-457)\end{array}$ & $\begin{array}{l}221 \\
(122-475)\end{array}$ & $\begin{array}{l}239 \\
(122-468)\end{array}$ & 0.529 \\
\hline T1000 & $\begin{array}{l}221.7 \\
(120-542)\end{array}$ & $\begin{array}{l}231.5 \\
(80-444)\end{array}$ & 0.412 & $\begin{array}{l}238.5 \\
(120-451)\end{array}$ & $\begin{array}{l}227 \\
(131-418)\end{array}$ & $\begin{array}{l}217.5 \\
(127-542)\end{array}$ & $\begin{array}{l}222 \\
(138-428)\end{array}$ & 0.418 \\
\hline T2000 & $\begin{array}{l}204.5 \\
(105-528)\end{array}$ & $\begin{array}{l}211 \\
(69-367)\end{array}$ & 0.530 & $\begin{array}{l}225 \\
(113-419)\end{array}$ & $\begin{array}{l}208 \\
(137-355)\end{array}$ & $\begin{array}{l}197 \\
(105-528)\end{array}$ & $\begin{array}{l}221 \\
(103-417)\end{array}$ & 0.687 \\
\hline
\end{tabular}

Data are presented as median $(\min -\max )$

$N$ nasal, $T$ temporal

${ }^{a}$ Wilcoxon sign test types on the basis of preoperative OCT findings: globally adherent membrane with diffuse edema, globally adherent membrane with intraretinal cystoid space, steepened foveal pit and/or lamellar split within the substance of the retina, and vitreomacular traction through a focal and adherent ERM. They suggested that postoperative outcome could be predicted according to this classification. Uji et al. [27] proposed that parallelism, which is the orientation of segmented lines in the SD-OCT image, is a novel marker for the diminishing structural integrity of retinal layers in eyes with ERM and an effective means of predicting visual acuity. Joe et al. [17] reported that the inner retinal layer thickness was the main predictor of visual acuity in eyes with ERM and classified ERM into three types according to the thickness of retinal layers. As a pathological mechanism of ERM-induced visual loss, they suggested that contractile forces induced by ERM cause inward movement of the outer nuclear layer, resulting in attachment of the adjacent parafoveal inner retinal layer that leads to disarrangement of the cells in each retinal layer and disturbance of normal neural transmission. Like other researchers who have conducted studies that support this pathological mechanism $[25,28]$, they found that the degree of inner retinal layer restoration with surgery was correlated with positive postsurgical visual outcome [17].

The latest staging system has been proposed by Govetto et al. [22]. It is a novel SD-OCT-based staging scheme for ERM, which takes into account the presence of continuous EIFL as a key finding associated with lower visual acuity in eyes with ERM. In this system, ERM is divided into four stages based on the findings on SD-OCT. As the stage of ERM increases, visual acuity significantly decreases. The presence of EIFL, a continuous hypo- or hyper-reflective band extending from the inner nuclear and inner plexiform layers across the foveal region, has been shown to be an independent risk factor for lower visual acuity [22]. Similarly, in our series, the visual acuity of eyes worsened with increasing stage of ERM, and on multivariate linear regression analysis, central foveal thickness and the presence of EIFL were independent predictors of visual acuity in eyes with ERM.

The development of EIFL has been suggested to result from the physical displacement of the inner retinal layers combined with Muller cell-driven proliferation due to tractional forces [29]. The correlation between the thickness of EIFL and visual acuity has been recently evaluated by Govetto et al. [30], who reported a negative correlation between the presence and thickness of EIFL and preoperative visual acuity. Similarly, in our study, eyes with EIFL were associated with significantly higher BCVA values. We also recorded that BCVA in $\log$ MAR was positively correlated with central foveal thickness $(r=$ $0.557, p<0.001)$ and EIFL thickness $(r=0.526, p<0.001)$, but not with outer nuclear layer thickness $(r=0.233, p=$ 0.123 ), which means that the visual acuity of eyes with ERM is negatively correlated with central foveal thickness and EIFL thickness. Therefore, we suggest that in addition to the presence of EIFL, its thickness may be evaluated for extent of ERM and timing of surgery.

In addition to EIFL, frequency of cystoid macular changes, ellipsoid disruption, and the cotton ball sign significantly increased from stage 1 through stage 4 in the study by Govetto et al. [22]. Similarly, in our study, the rate of cystoid changes, cotton ball sign, and disruption of the ellipsoid zone increased with increasing stage of ERM. However, whereas neither the presence of cystoid changes nor the cotton ball sign had a significant effect on BCVA, disruption of the ellipsoid zone was associated with significantly higher BCVA values in logMAR. On the other hand, none of these SD-OCT findings were found to be an 
independent predictor of visual acuity in a multivariate linear regression model. In eyes with ERM, disruption of the ellipsoid zone has been reported to be negatively correlated with visual outcomes in multiple studies [14, 31]. However, in our study, we did not find a correlation between disruption of the ellipsoid zone and visual acuity, probably due to the small number of patients with ellipsoid zone disruption.

Arichika et al. [11] showed that although foveal retinal thickening was significantly greater at the inner than in the outer layer in ERM, only outer retinal thickening was significantly correlated with visual acuity. On the contrary, although outer nuclear layer thickness changed as the stage of ERM progressed in our study, it was not correlated with visual acuity and was not a significant predictive factor for visual acuity in eyes with ERM in the multivariate linear regression in our study. This difference may be due to the automated segmentation performed by the SD-OCT instrument pointed out by Arichika et al. [11], which prevents the optimal measurement of retinal layers.

Lower central foveal thickness, a thinner ganglion cellinner plexiform layer, and a higher central foveal thickness/ ganglion cell layer ratio were reported as significant prognostic factors for visual improvement after ERM surgery [31, 32]. Decreased vision in eyes with ERM has been attributed to the thickening of the inner foveal retinal layer and central foveal thickness, which have also been found to be predictors of poor visual outcome and metamorphopsia after ERM surgery [33, 34]. Other prognostic factors for surgical outcome in eyes with ERM include age, duration of disease, baseline BCVA, inner and outer segment junction disruption, and inner-retinal irregularity [35, 36]. Govetto et al. [30] recently suggested that the presence of EIFL is a negative prognostic factor for postoperative anatomical and functional recovery. We did not evaluate the timing and indication for surgical treatment of ERM or the postoperative visual outcome in our patients. To confirm the results of Govetto et al. [30], further studies should address the predictive value of staging of eyes with ERM and the value of measuring the presence and thickness of EIFL to predict the postoperative visual outcome.

There are limited and conflicting studies on the choroidal thickness and structure in eyes with ERM [20, 21, 37]. Because choroidal abnormalities play a key role in major posterior segment ocular diseases, choridal thickness and structure may be expected to change in eyes with ERM. Due to tractional forces in eyes with ERM, retinal arteries become dilated and tortuous, which may affect the hemodynamics and thickness of the choroid [38, 39]. Some studies have indicated a temporary change in choroidal thickness after vitrectomy of eyes with ERM and suggested that there is a relationship between choroidal thickness and the presence of ERM $[37,38]$. On the other hand, Casini et al. [39] recently reported that choroidal thickness reduction is probably due to the exposition of ocular tissues to a more highly oxygenated environment (vitrectomy effect) and constriction of the choroidal vessels after vitrectomy of eyes with ERM. Several studies have also revealed that eyes with ERM and fellow eyes without ERM did not show any significant difference in terms of choroidal thickness at baseline [37-40]. In our study, we found similar results: no significant difference in terms of choroidal thickness between eyes with ERM and fellow unaffected eyes. In addition, we are the first researchers to reveal that with increasing stage of ERM and increasing level of contraction, choroidal thickness did not change significantly between different stages of ERM within eyes. Therefore, our findings imply that choroidal structure was not influenced by the development and progression of ERM.

The main limitation of the present study was its crosssectional design, which prevents the evaluation of the postoperative visual outcome of ERM and prognostic factors. Furthermore, subjective interpretation of SD-OCT findings is a drawback that needs to be noted, which may limit the standard use of the SD-OCT-based staging system. Nevertheless, this study offers important insights into the novel SD-OCT-based staging system of ERM in clinical practice, and it demonstrates that the presence and thickness of EIFL are inversely correlated with the visual acuity of eyes with ERM.

In conclusion, staging of ERM by SD-OCT according to the presence of EIFL is effective for grading retinal damage and visual loss in eyes with ERM. The presence and thickness of EIFL and central foveal thickness are key indicators of visual acuity loss in eyes with ERM. In addition, our study revealed that the development and progression of ERM are not associated with choroidal thickness. Further prospective clinical studies with larger sample sizes should address the criteria for indications and timing of surgery and the relationship between different stages of ERM and visual prognosis. Additionally, clinicopathological and electrophysiological investigations are needed to better understand the pathophysiology of EIFL.

\section{Summary}

\section{What was known before}

- We aimed to evaluate the relationship between the presence of ectopic inner foveal layers (EIFL), microstructural changes of the macula, choroidal thicknesses, and visual acuity in patients with epiretinal membranes (ERM) in different stages defined by spectral-domain optical coherence tomography (SD-OCT). 


\section{What this study adds}

- The SD-OCT staging according to the presence of EIFL is effective for grading retinal damage, macular microstructural changes, and visual loss in eyes with ERM.

- Presence and thickness of EIFL, and central foveal thickness are key indicators of visual acuity loss in ERM.

\section{Compliance with ethical standards}

Conflict of interest The authors declare that they have no conflict of interest.

\section{References}

1. Fraser-Bell S, Guzowski M, Rochtchina E, Wang JJ, Mitchell P. Five-year cumulative incidence and progression of epiretinal membranes: the Blue Mountains Eye Study. Ophthalmology. 2003;110:34-40.

2. Fraser-Bell S, Ying-Lai M, Klein R, Varma R. The Los Angeles Latino Eye Study Group. Prevalence and associations of epiretinal membranes in Latinos: the Los Angeles Latino Eye Study. Invest Ophthalmol Vis Sci. 2004;45:1732-6.

3. McCarty DJ, Mukesh BN, Chikani V, Wang JJ, Mitchell P, Taylor $\mathrm{HR}$, et al. Prevalence and associations of epiretinal membranes in the visual impairment project. Am J Ophthalmol. 2005; 140:288-94.

4. Ng CH, Cheung N, Wang JJ, Islam AF, Kawasaki R, Meuer SM, et al. Prevalence and risk factors for epiretinal membranes in a multi - ethnic United States population. Ophthalmology. 2011;118:694-9.

5. Jahn CE, Minich V, Moldaschel S, Stahl B, Jedelhauser P, Kremer $\mathrm{G}$, et al. Epiretinal membranes after extracapsular cataract surgery. J Cataract Refract Surg. 2001;27:753-60.

6. Mori K, Gehlbach PL, Sano A, Deguchi T, Yoneya S. Comparison of epiretinal membranes of differing pathogenesis using optical coherence tomography. Retina. 2004;24:57-62.

7. Council MD, Shah GK, Lee HC, Sharma S. Visual outcomes and complications of epiretinal membrane removal secondary to rhegmatogenous retinal detachment. Ophthalmology. 2005; 112:1218-21.

8. Gass JDM. Macular dysfunction caused by epiretinal membrane contraction. In: Stereoscopic atlas of macular diseases: diagnosis and treatment. Vol. 2, 4th ed. St Louis, MO: Mosby; 1997, p. 938-950.

9. Koizumi H, Spaide RF, Fisher YL, Freund KB, Klancnik JM Jr, Yannuzzi LA. Three-dimensional evaluation of vitreomacular traction and epiretinal membrane using spectral-domain optical coherence tomography. Am J Ophthalmol. 2008; 145:509-17.

10. Michalewski J, Michalewska Z, Cisiecki S, Nawrocki J. Morphologically functional correlations of macular pathology connected with epiretinal membrane formation in spectral optical coherence tomography (SOCT). Graefes Arch Clin Exp Ophthalmol. 2007;245:1623-31.

11. Arichika S, Hangai M, Yoshimura N. Correlation between thickening of the inner and outer retina and visual acuity in patients with epiretinal membrane. Retina. 2010;30:503-8.
12. Kim JH, Kim YM, Chung EJ, Lee SY, Koh HJ. Structural and functional predictors of visual outcome of epiretinal membrane surgery. Am J Ophthalmol. 2012;153:103-10.

13. Shimozono M, Oishi A, Hata M, Matsuki T, Ito S, Ishida K, et al. The significance of cone outer segment tips as a prognostic factor in epiretinal membrane surgery. Am J Ophthalmol. 2012;153:698-704.

14. Itoh Y, Inoue M, Rii T, Hirota K, Hirakata A. Correlation between foveal cone outer segment tips line and visual recovery after epiretinal membrane surgery. Invest Ophthalmol Vis Sci. 2013;54:7302-8.

15. Watanabe K, Tsunoda K, Mizuno Y, Akiyama K, Noda T. Outer retinal morphology and visual function in patients with idiopathic epiretinal membrane. JAMA Ophthalmol. 2013;131:172-7.

16. Okamoto F, Sugiura Y, Okamoto Y, Hiraoka T, Oshika T. Time course of changes in aniseikonia and foveal microstructure after vitrectomy for epiretinal membrane. Ophthalmology. 2014; 121:2255-60.

17. Joe SG, Lee KS, Lee JY, Hwang JU, Kim JG, Yoon YH. Inner retinal layer thickness is the major determinant of visual acuity in patients with idiopathic epiretinal membrane. Acta Ophthalmol. 2013;91:e242-3.

18. Lee EK, Yu HG. Ganglion cell-inner plexiform layer thickness after epiretinal membrane surgery: a spectral-domain optical coherence tomography study. Ophthalmology. 2014; 121:1579-87.

19. Vieira LM, Silva NF, Dias dos Santos AM, dos Anjos RS, Pinto LA, Vicente AR, et al. Retinal ganglion cell layer analysis by optical coherence tomography in toxic and nutritional optic neuropathy. J Neuroophthalmol. 2015;35:242-5.

20. Michalewska Z, Michalewski J, Ornafel-Sagan K, Nawrocki J. Swept-source optical coherence tomography correlations between retina and choroid before and after vitrectomy for epiretinal membranes. Am J Ophthalmol. 2016;165:100-7.

21. Kang EC, Lee KH, Koh HJ. Changes in choroidal thickness after vitrectomy for epiretinal membrane combined with vitreomacular traction. Acta Ophthalmol. 2017;95:e393-8.

22. Govetto A, Lalane RA 3rd, Sarraf D, Figueroa MS, Hubschman JP. Insights into epiretinal membranes: presence of ectopic inner foveal layers and a new optical coherence tomography staging scheme. Am J Ophthalmol. 2017;175:99-113.

23. Tsunoda K, Watanabe K, Akiyama K, Usui T, Noda T. Highly reflective foveal region in optical coherence tomography in eyes with vitreomacular traction or epiretinal membrane. Ophthalmology. 2012;119:581-7.

24. You Q, Xu L, Jonas JB. Prevalence and associations of epiretinal membranes in adult Chinese: the Beijing eye study. Eye. 2008;22:874-9.

25. Hwang JU, Sohn J, Moon BG, Joe SG, Lee JY, Kim JG, et al. Assessment of macular function for idiopathic epiretinal membranes classified by spectral-domain optical coherence tomography. Invest Ophthalmol Vis Sci. 2012;53:3562-9.

26. Kinoshita T, Kovacs KD, Wagley S, Arroyo JG. Morphologic differences in epiretinal membranes on ocular coherence tomography as a predictive factor for surgical outcome. Retina. 2011;31:1692-8.

27. Uji A, Murakami T, Unoki N, Ogino K, Nishijima K, Yoshitake $\mathrm{S}$, et al. Parallelism as a novel marker for structural integrity of retinal layers in optical coherence tomographic images in eyes with epiretinal membrane. Am J Ophthalmol. 2014;157:227-36.

28. Yoon YH, Joe SG, Hwang JU, Yang HS. Insights into epiretinal membranes: presence of ectopic inner foveal layers and a new optical coherence tomography staging scheme. Am J Ophthalmol. 2017;177:226-7.

29. Govetto A, Su D, Farajzadeh M, Megerdichian A, Platner E, Ducournau $\mathrm{Y}$, et al. Microcystoid macular changes in association 
with idiopathic epiretinal membranes in eyes with and without glaucoma: clinical insights. Am J Ophthalmol. 2017;181:156-65.

30. Govetto A, Virgili G, Rodriguez FJ, Figueroa MS, Sarraf D, Hubschman JP. Functional and anatomical significance of the ectopic inner foveal layers in eyes with idiopathic epiretinal membranes: surgical results at 12 months. Retina. 2017. https:// doi.org/10.1097/IAE.0000000000001940.

31. Miguel AI, Legris A. Prognostic factors of epiretinal membranes: a systematic review. J Fr Ophtalmol. 2017;40:61-79.

32. Song SJ, Lee MY, Smiddy WE. Ganglion cell layer thickness and visual improvement after epiretinal membrane surgery. Retina. 2016;36:305-10.

33. Yang HS, Kim JT, Joe SG, Lee JY, Yoon YH. Postoperative restoration of foveal inner retinal configuration in patients with epiretinal membrane and abnormally thick inner retina. Retina. 2015;35:111-9.

34. Okamoto F, Sugiura Y, Okamoto Y, Hiraoka T, Oshika T. Inner nuclear layer thickness as a prognostic factor for metamorphopsia after epiretinal membrane surgery. Retina. 2015;35:2107-14.

35. Cho KH, Park SJ, Cho JH, Woo SJ, Park KH. Inner-retinal irregularity index predicts postoperative visual prognosis in idiopathic epiretinal membrane. Am J Ophthalmol. 2016;168:139-49.
36. Kauffmann Y, Ramel JC, Lefebvre A, Isaico R, De Lazzer A, Bonnabel A, et al. Preoperative prognostic factors and predictive score in patients operated on for combined cataract and idiopathic epiretinal membrane. Am J Ophthalmol. 2015;160:185-92.

37. Ahn SJ, Woo SJ, Park KH. Choroidal thickness change following vitrectomy in idiopathic epiretinal membrane and macular hole. Graefes Arch Clin Exp Ophthalmol. 2016;254:1059-67.

38. Michalewska Z, Michalewski J, Adelman RA, Zawiślak E, Nawrocki J. Choroidal thickness measured with swept source optical coherence tomography before and after vitrectomy with internal limiting membrane peeling for idiopathic epiretinal membranes. Retina. 2015;35:487-91.

39. Casini G, Loiudice P, Lazzeri S, Pellegrini M, Ripandelli G, Figus $\mathrm{M}$, et al. Analysis of choroidal thickness change after 25-gauge vitrectomy for idiopathic epiretinal membrane with or without phacoemulsification and intraocular lens implantation. Ophthalmologica. 2017;237:78-84.

40. Maul EA, Friedman DS, Chang DS, Boland MV, Ramulu PY, Jampel HD, et al. Choroidal thickness measured by spectral domain optical coherence tomography: factors affecting thickness in glaucoma patients. Ophthalmology. 2011;118:1571-9. 